\title{
Structure, function and assembly of the long, flexible tail of siphophages
}

\author{
Romain Linares ${ }^{1,3}$, Charles-Adrien Arnaud ${ }^{2,3}$, Séraphine Degroux ${ }^{1}$, Guy Schoehn ${ }^{1}$ and \\ Cécile Breyton ${ }^{1}$ \\ ${ }^{1}$ Univ. Grenoble Alpes, CNRS, CEA, Institute for Structural Biology, F-38000 Grenoble, France \\ ${ }^{2}$ Hockmeyer Hall of Structural Biology, Purdue University, West Lafayette, IN 47907, USA \\ Corresponding author: Cécile Breyton (Cecile.Breyton@ibs.fr) \\ ${ }^{3}$ These authors contributed equally to this work.
}

\begin{abstract}
Bacteriophages, viruses that infect bacteria, are the most abundant biological entities on Earth. Siphophages, accounting for $\sim 60 \%$ of known phages, bear a long, flexible tail that allows host recognition and safe delivery of the DNA from the capsid to the cytoplasm of the infected cell. Independently from their host (Gram positive or Gram negative) and the nature of their receptor at its surface (polysaccharide or protein), the core tail architecture of all caudophages and of phagederived systems share the same structural organisation and are thought to be homologous. Here, we review the recent advances in the structure, function and assembly of the core tail architecture of siphophages.
\end{abstract}

\section{Introduction}

The vast majority of known bacterial viruses are tailed bacteriophages. They consist of a capsid containing a densely packed double-stranded DNA and a tail. Depending on the morphology of their tail, phages are classified as Siphoviridae (long flexible tail), Myoviridae (long contractile tail) and Podoviridiae (short tail). The assembly pathway of the capsid and of the long tail are independent: DNA-full capsids and assembled tails connect to form the complete virion, which is liberated with cell lysis. Because its interaction with the cell surface triggers host infection, the phage tail is an extremely interesting study subject in terms of its assembly pathway, structure, host recognition and cell wall perforation mechanisms. Indeed, the tail serves to recognise the host and safely deliver the genome into the bacterial cytoplasm. Thus, at its distal extremity, the tail tip complex is equipped with Receptor Binding Proteins (RBPs), which are present in one to several copies (up to e.g. 54 in siphophage 2 and 72 in myophage CBA120). In siphophages, optional side tail fibres may also be present. The core of the tip complex is formed by a ring of the hexameric Distal Tail Protein (DTP) and a trimeric ring of the Baseplate Hub Protein (BHP). RBPs are attached either via the DTP or at the extremity of a central fibre that is attached to the BHP (Fig. 1A). On the proximal side of the tip complex, the tail continues as a long tube, formed by the oligomerisation of the Tail Tube Protein (TTP) around the Tape Measure Protein (TMP). The tube ends with the Terminator Protein and in some cases the Tail Completion Protein (Fig. 1A). In myophages, the tail tube is enveloped by the sheath. Whereas the primary sequences of structural tail proteins of siphoand myo-phages have diverged and share very low sequence similarity, the relative position of genes coding for tail structural proteins in phage genomes is remarkably conserved (Fig. 1B), pointing to a common ancestor. This hypothesis is further consolidated by the striking structural similarity within phage tail proteins [1]. An extensive review that summarises many decades of research and that covers a broad range of topics concerning the non-contractile tails of siphophages was published in 2012 [2]. The present short review is an update, highlighting the work that has been published since then, focusing on the structure, function and assembly of the non-contractile, flexible phage tails.

\section{The proximal extremity of siphophage tails}

As mentioned above, full capsids and assembled tails come together to form the complete phage particle. It was shown, for SPP1, that on the tail side, this "glue" function is performed by the terminator protein [3]. The terminator protein is a highly conserved protein within long-tailed 
phages $[2,4,5]$ (Linares et al., in preparation) that terminates the tail tube, making a final hexameric ring after the last TTP hexamer [6]. The same fold, reminiscent of the TTP fold, is conserved in myophages to terminate not only the tail tube, but also the sheath [7].

The tail completion protein, product of another highly conserved gene, is believed to be present at the proximal end of the siphotail, possibly to help correct positioning of the DNA after capsid-tail joining. No further information is available other than its detection in T5 virions and purified tails [4], but it was not identified in the structure of the head-to-tail interface of SPP1 [6]. For an interesting head-to-tail architecture discussion, see [8,9].

\section{The Tail Tube Protein (TTP)}

The flexible tail tube is formed by the stacking of hexameric rings of the TTP (also called Major Tail Protein). Its structure consists of a sandwich of 2 antiparallel $\beta$ sheets, an $\alpha$ helix on one side and a long loop [10], and upon polymerization, the fold becomes more structured [11-13]. This fold is shared by the terminator protein, the DTP and the BHP of siphophages (Fig. 2), but also of the homologous proteins of myophages and related contractile injection systems [14]. Interestingly in T5, the TTP results in gene duplication and fusion, and the tail tube results in the stacking of trimeric rings of the TTP (Fig. 2,3A)[12].

Far from being just a passive architecture linking the capsid to the tip complex, the tail tube has multiple functions. TTP are commonly observed to self-assemble in vitro as seen with SPP1gp17 [15], T5-pb6 [12] and vB_EcoS_NBD2-gp39 [16]. In vivo however, tail assembly is an extremely regulated process, and "wild" self-assembly does not seem to occur. Whether assembly of the tail tube is driven by self-assembly properties or not remains to be determined. The tail tube contains the TMP in a metastable state (see below) and facilitates transfer of DNA, with its negatively charged lumen surface, as seen for T5 and $80 \alpha$ (Fig. 3B). Interestingly, it does not seem to be the case for $\lambda$ [13], maybe because of its viral entry requiring larger amount of the host energy-driven machinery. This will need however to be confirmed with higher resolution data.

The tail tube of Siphoviridae had also been suggested to carry out a role of signal transmission leading to the opening of the capsid and DNA release upon receptor binding, through TTP conformational changes [17]. However this hypothesis was based on negative stain, low resolution data. Since then, higher-resolution structures of T5 [12] and $\lambda[13]$ tubes have been solved pre- and post-interaction with the host receptor, revealing no conformational changes of the TTP. Thus, it is now hypothesised that DNA release is triggered by TMP release (see §TMP). Capsid opening would occur when tail and capsid assemble or upon host binding. Note that these two hypotheses are not fully exclusive and may furthermore be extended to Myoviridae. Although the fold conservation of core building blocks of the tail advocates for a common mechanism, different phages might have adopted different strategies. A deeper knowledge of the head-to-tail interfaces will yield answers on this missing step of the viral assembly and entry.

In siphophages, the TTP is free to carry 'decoration' domains. Ig-like domains of the Big2, I-set and FN3 families are commonly found as C-terminal extensions to Caudovirales structural proteins, and especially TTPs [18]. These domains are thought to enhance infectivity through weak interactions with the cell wall. In tail tubes, they are arranged in different fashions along the tube: T5 TTP has three domains per ring, sticking out tangentially to the TTP ring and perpendicular to the tube axis, whereas the six domains per ring are arranged parallel to the tube axis in $\lambda$ (Fig. 3A); lactophage 1358 has three consecutive domains on each TTP, but p2 and $80 \alpha$ have none (Fig. 1B,3A). In SPP1, the TTP gene yields two proteins through a programmed translational frameshifting, with or without a C-terminal Ig-like domain, in a 3:1 ratio (Fig. 1B) $[15,19]$.

In T5, the first tube ring after the DTP is formed by a minor tail protein, $\mathrm{p} 140$, which has a similar fold as the TTP but lacks the decoration domain. It is instead covered by the 'collar', a dodecameric ring to which are attached the side fibres, making p140 a bona fide member of the tip complex (Fig. 1B, 2)(Linares et al., in preparation). This is reminiscent of myophages, which also bear a modified TTP ring within their baseplate [14].

Decoration domains could also be involved in the mechanical qualities of the tube: interactions between the Ig-domain and the T5 tube were reported [20] and in SPP1, the absence of the Ig-like domain affects tail flexibility [19]. In phage $80 \alpha$, which lacks the TTP decoration domain, the TTP C-terminus makes additional inter-ring interactions, which could indicate that the lack of Ig-like domains would need to be compensated (Fig. 3A)[21]. A C-terminal extension is also observed in p2 TTP, which also lacks a decoration domain. 
Unlike those of contractile systems, tubes of Siphoviridae are not required to be rigid to perforate the cell wall. Flexibility of siphophages tubes was attributed to a difference in N-terminus and other loops, making more extensive inter-ring interactions in rigid tube assemblies $[12,13]$. Modulation of tail flexibility and its impact on particles infectivity could prove an interesting field of study in the near future.

\section{The Tape Measure Protein (TMP) and the Tail Associated Chaperones (TACs)}

As its name suggests, the TMP determines the length of the tail tube, both in Siphoviridae and in Myoviridae, as elegantly demonstrated on $\lambda$ [22], and more recently on TP901-1 [23]. These latter authors further dissect the requirement of the different domains of the protein. No structure of TMP is yet available, but it is proposed to be located, in a stoichiometry of $\sim 6$ and as a helix bundle or coiled-coil, in the lumen on the tail tube, with its $\mathrm{N}$-terminus at the proximal end of the tail, and its C-terminus at the distal end. In the C-terminal sequence, hydrophobic segments and enzymatic domains involved in peptidoglycan hydrolysis can be identified (e.g. [24]). In several phages, a fragment of the C-terminus is cleaved $($ e.g. $[4,25])$, but whether this is a general trait has not been systematically investigated. In a very recent siphophage tip structure, the last 21 amino acids of the TMP of phage $80 \alpha$ were solved, as a trimer [21]. In our own structure of phage T5 tip, we also see three copies of the $35 \mathrm{C}$-terminal residues of the TMP. These are the C-terminus of the full-length protein, showing that the small proteolysed C-terminal fragment is retained in the tail tube. Mass spectrometry analysis shows that this fragment includes the peptidoglycan hydrolase domain of the protein (Linares et al., in preparation), suggesting that this domain needs to be independent from the rest of the protein during cell wall perforation. Interestingly, both in $80 \alpha$ and T5, only the last C-terminal residues are resolved, because of specific interactions with the BHP. Densities belonging to the rest of the TMP are present but is uninterpretable in terms of residues. This could be due to the low/a-specific/no interaction of the TMP with the inner surface of the tail tube.

During tail assembly, the long TMP is stabilised by chaperones. In the majority of phages, two proteins, named from $\lambda \mathrm{G}$ and GT, are synthesised, via a programmed translational -1 frameshift. Efficacy of frameshift determines the G/GT ratio, which has been shown to be crucial for correct tail assembly, as well as the covalent link between G and T in the GT protein [26]. Structural analyses suggest that G coats the TMP as a spiral, preventing TMP aggregation, with occasional insertions of GT in the G spiral. Indeed, despite sequence and oligomerisation mode divergence, the spiral quaternary structure, which would stabilise the TMP without the need for sequence recognition, seems to have been conserved $[27,28]$. In $\lambda$, TTP polymerisation around the TMP was shown to be initiated by the GT-TTP interaction, which would recruit and convert the TTP into an assembly competent conformation, acting as an oligomerisation nucleus [28]. It was proposed that the TMP is folded in the tail tube in a metastable conformation, which would relax, after host binding, into a conformation of lesser energy inducing expulsion from the tail after host binding [12] (reminiscent of the metastable sheath fold of Myoviridae and of the unfolding/refolding of core proteins of Podoviridae). Expulsion of the TMP from the tube would trigger DNA release from the capsid. Proteolysis of the C-terminus could induce a transition from a stable chaperone-bound state, to a metastable TTP-bound state. Supporting this hypothesis is the fact that in $\lambda$, proteolysis occurs only after tail assembly is complete [25], and the observation that purified tails are not stable: the first sign of aging in T5 tails is the release of the TMP from the proximal end of the tail.

Due to space limitation, we will not discuss in details the fate of the TMP after expulsion. In a nutshell: the TMP is proposed to form the channel that could span the membrane(s) [23,24], but spanning of the inner membrane would require interaction with a host protein [29]. Furthermore, in HK97 and TP-J34, the TMP is the target of the Superinfection Exclusion protein, protecting lysogens from over-infection $[29,30]$.

\section{The Tail Tip Complex}

Located at the distal end of the tail, the tip complex is the host-recognition apparatus of the phage. Although very diverse in size and shape, from large baseplate-like structures to very pareddown ones, the tip comprise two structurally conserved proteins, the BHP (or Tal) and the DTP (or Dit) that are also the core of myophages and bacterial phage-related injection systems [14]. The tip complex serves as a hub for RBPs binding, either directly or indirectly (via e.g. TP901-1-BppU [31] or Tuc2009-BppA [32]) for polysaccharide binding RBPs, or at the extremity of the central fibre. 
Over the past decade, several siphophage tip structures, or parts thereof, have become available, often by combination of EM and X-ray crystallography, and more recently with high-resolution cryo-EM, both for Gram-positive (SPP1 [33], TP901-1 [34], p2, [35], Tuc2009 [32] and 80 $\alpha$ [21]) and Gram-negative infecting phage T5 [Linares et al., in preparation]. Tail assembly has been extensively studied for phage $\lambda$. Recent studies on phages p2 and TP901-1 confirm the central role of DTP/BHP oligomerisation and assembly, together with the TMP/TAC complex, which seems to be the initiator complex. Assembly of the initiator complex is followed by polymerisation of the TTP to form a tube, and the attachment of peripheral proteins to the baseplate [36-38].

\section{The Distal Tail Protein (DTP)}

The DTP is located between the first TTP ring and the BHP trimer and assembles as a hexameric ring continuing the tail tube (Fig. 1A,2,4A). In a remarkable paper from 2010, two back to back hexamers have been suggested in $\mathrm{p} 2$, from fitting the baseplate crystal structure in a cryoEM map of the virion at $22 \AA$ resolution [35]. In view of the extremely concerved core of the baseplate, this seems however unlikely. Previously thought to be specific to Gram-positive infecting siphophages, it appears now clearly that DTPs are a widespread building block also shared by Gram-negative infecting siphophages [39] and myophages [14]. DTPs exhibit a TTP fold, even though sequence is very poorly conserved, to which decoration domains can be grafted (Fig. $4 \mathrm{~A})[21,33,34,39]$, but this is not always the case [39,40]. Gram-positive siphophages often exhibit a galectin domain, inserted at the C-terminal extremity of the TTP domain [41], whereas an oligosaccharide/oligonucleotide-binding fold domain is found in Gram-negative phage T5, inserted in a loop of the TTP domain [39](Fig. 4A). "Evolved DTPs" have also been reported to exhibit domain insertions [42], able to act as bona fide RBPs [43,44]. All these decoration domains share oligosaccharide binding properties and contribute, as TTP Ig-like domains, to unspecific cell wall binding. In "evolved DTPs" however, these domains are directed against the same polysaccharides as the RBPs, suggesting co-evolution to adapt to the same host-specific cell wall associated polysaccharide type [42].

\section{The Baseplate Hub Protein (BHP)}

The BHP assembles as a trimer below the DTP ring (Fig. 1A, 2,4B). Although being very variable in length, sipho- and myophage BHPs share a four "Hub Domain" (HD) common scaffold. HDI and III appear to be structurally very conserved and consist each of a TTP fold, continuing the tail tube with a pseudo 6-fold symmetry. It thus acts as an adaptor between the upper DTP hexamer and the optional trimeric central fibre (Fig. 2). In siphophages, HDII and IV form the distal part of the BHP, which closes the tail tube (Fig. 2,4B). This is different from myophages and phage-related injection systems, in which the tail tube is closed by the central membrane piercing needle (gp5like) $[14,45]$.

BHP can also form partially (e.g. T5 [Linares et al., in preparation]) or probably completely ( $\lambda$ and SPP1 $[33,46])$ the central fibre, which can partly be composed of fibronectin domains (Fig. $1 \mathrm{~B}, 2,4 \mathrm{~B}$ )[Linares et al., in preparation]. In $\lambda$, the BHP is formed by several proteins: gpL is related to HDI and gpJ to HDIII-IV. GpK and gpI, although located between these two proteins in the morphogenetic block, appear unrelated to BHP HDII, but gpI could serve the function, considering its gene position in the genome.

Other decorative/accessory domains can also be found in BHP: C-terminal domains with peptidoglycan hydrolase enzymatic activities are reported, suggesting a role in cell wall degradation and which first gave them the name "Tail Associate Lysozyme" (Tal) [1,36,40,47] (Fig. 1B). In Tuc2009 and TP901-1, these domains can be removed by proteolysis during morphogenesis, leading to two phage populations: one, without the hydrolase domain, with enhanced host-binding capacities, while the other one, bearing the hydrolase domain, is able to infect cell in stationary phase, for optimized infection [48]. Other decorative domains, with putative carbohydrate-binding activities, have also been reported [43].

Following interaction with the receptor, the HDII-IV domains widen, opening the tail tube. This was suggested by the different conformations of SPPI and 1358 baseplate and tip low resolution structures $[33,49]$ and directly confirmed, following receptor interaction, on T5 tip [Linares et al., in preparation]. The opening of the BHP would destabilise interactions with the TMP, liberating it and committing the phage to infection. 


\section{The Receptor Binding Proteins (RBPs) and Side Fibre Proteins}

The size limit of the review does not permit us to detail this aspect. RBPs represent the most divergent proteins in phage tails, and different strategies and protein have been developed to adapt to the very different cell surface of hosts. Very good reviews, concerning mostly Gram-positive recognition by phages, have been recently published [49-53].

\section{Concluding remarks}

With the wealth of phage protein structures and more recently, with the "resolution revolution" of cryo-EM, of entire phages, and the always more powerful in silico prediction tools, it has become clear that phages share much more than previously thought. Indeed, despite great sequence diversity, we can but acknowledge great structure conservation. A common building block, the TTP fold, is decorated with different accessory domains to serve different functions and interact with different partners, to finally form the tail. This original module is not only common to all the proteins of the siphotail tube (Fig. 2), but is also shared by the myotail proteins and all the bacterial phage-derived injection system tube protein. It appears the same fold is also shared by podophage knob proteins [54], and maybe even by non-structural proteins [55]!

However, much is still unclear. The chain of events and conformational changes, starting with RBP interaction with its bacterial receptor and resulting in cell wall perforation and DNA ejection, are still not understood; the formation and structure of the channel spanning the bacterial envelope, the interaction with host factors, are also of particular interest, and start to become accessible, e.g. by cryo-electron tomography [56,57], promising fascinating years to come in the study of phage structural biology.

\section{Acknowledgments}

We thank Paulo Tavares and Rudi Lurz for the image of SPP1 for the graphical abstract. This work acknowledges funding from the Grenoble Instruct-ERIC center (ISBG; UMS 3518 CNRSCEA-UGA-EMBL) within the Grenoble Partnership for Structural Biology (PSB), supported by FRISBI (ANR-10-INBS-05-02) and GRAL, financed within the University Grenoble Alpes graduate school (Ecoles Universitaires de Recherche) CBH-EUR-GS (ANR-17-EURE-0003), and from the grant ANR-16-CE11-0027-01. IBS acknowledges integration into the Interdisciplinary Research Institute of Grenoble (IRIG, CEA). 


\section{Figures}
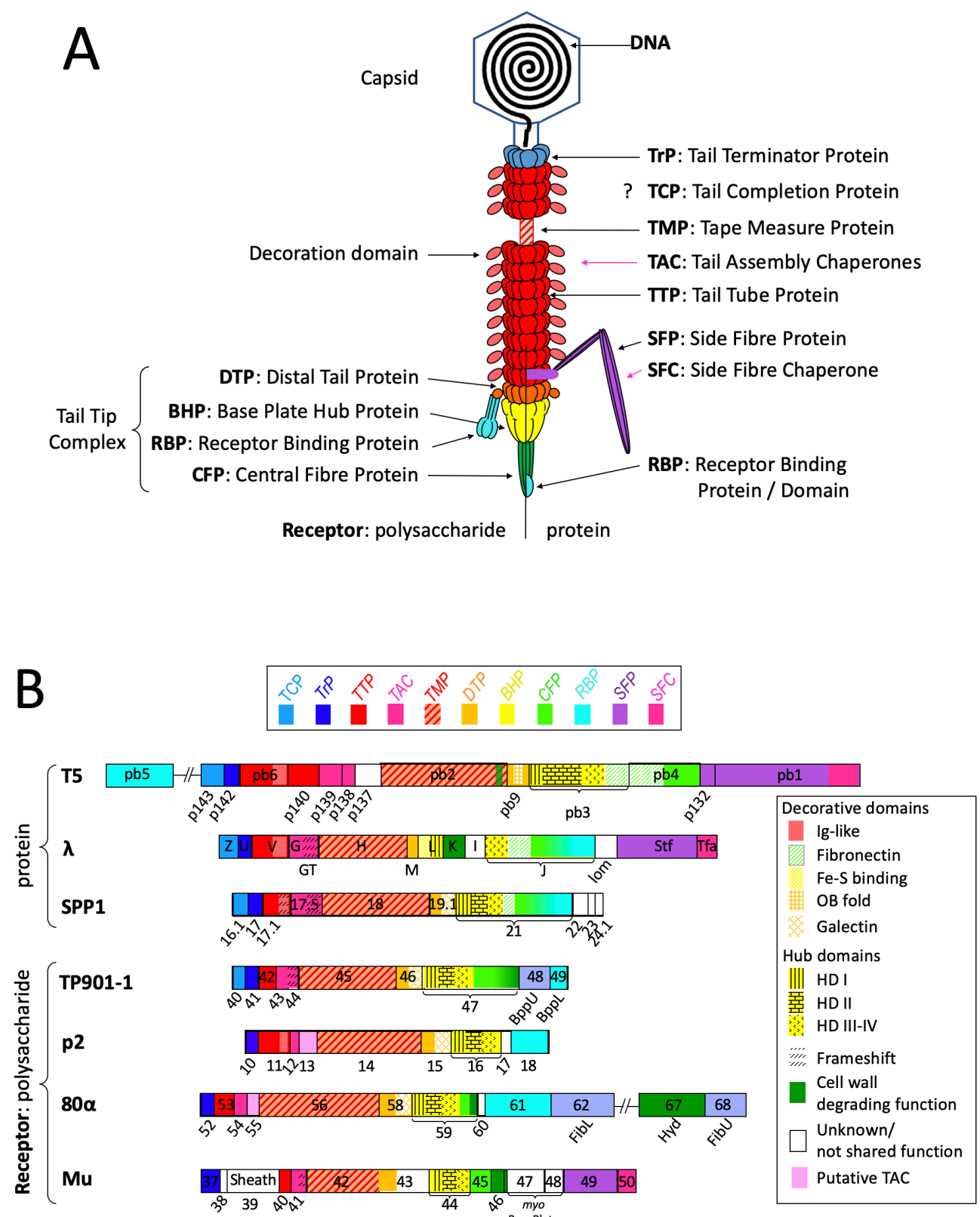

Figure 1: A. Schematic representation of siphophages. Conserved tail protein are indicated. The position of the tail completion protein is not known. The pink arrows indicate chaperones. TTP and BTP proteins do not always show decoration domains. When the receptor is a polysaccharide, RBPs are present in multiple copies and bound directly or indirectly to the DTP, and the central fibre protein can be absent (left). When the phage targets a protein as main receptor, the RBP is located at the end of the central fibre, as an individual protein or a domain of the central fibre protein (right). The side fibres are not always present. The colour code is the same as that of panel B. Terminator protein, TTP, DTP, BHP HDI-I and HDIII share the same fold, represented by the apple-like shape. The capsid and the tail are not on the same scale. B. General arrangement of siphophage tail genes. The region encoding tail proteins is shown for $E$. coli siphophages $\lambda$ and T5, B. subtilis siphophage SPP1, L. lactis siphophages TP901-1 and p2, S. aureus siphophage $80 \alpha$, as well as for E. coli myophage Mu. Genes encoding proteins of homologous function/structure are shown in the same colour. Abbreviation are that of panel A. Similarities were established by structural data and through HHpred searches. No frameshift consensus sequences were found in neither T5 nor $80 \alpha$ TAC gene (but an overlap with the following ORF is possible in both cases); one is clearly identified in $\mathrm{p} 2 \mathrm{TAC}$, but there is no possible overlap with the following ORF. 


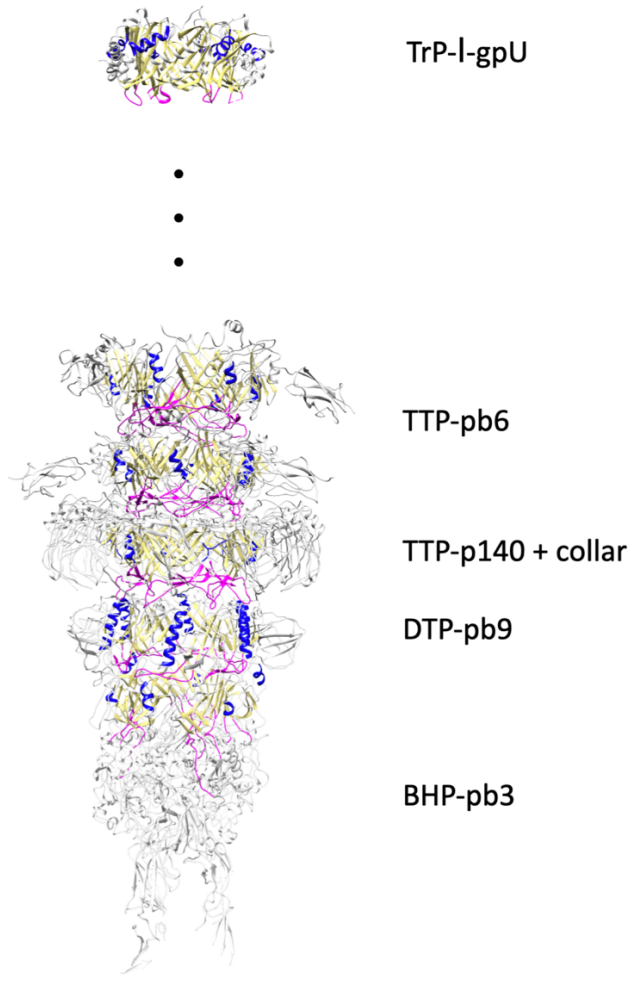

Figure 2: Structure of the tail tip of T5, highlighting the common features of the TTP fold in the different tail proteins. The side helix is coloured blue, the beta sandwich, pale yellow and the large loop, magenta (from Linares et al., in preparation). The terminator protein, reminiscent of the TTP, is that of $\lambda(3 F Z 2)$.

TTP-p140 + collar

DTP-pb9
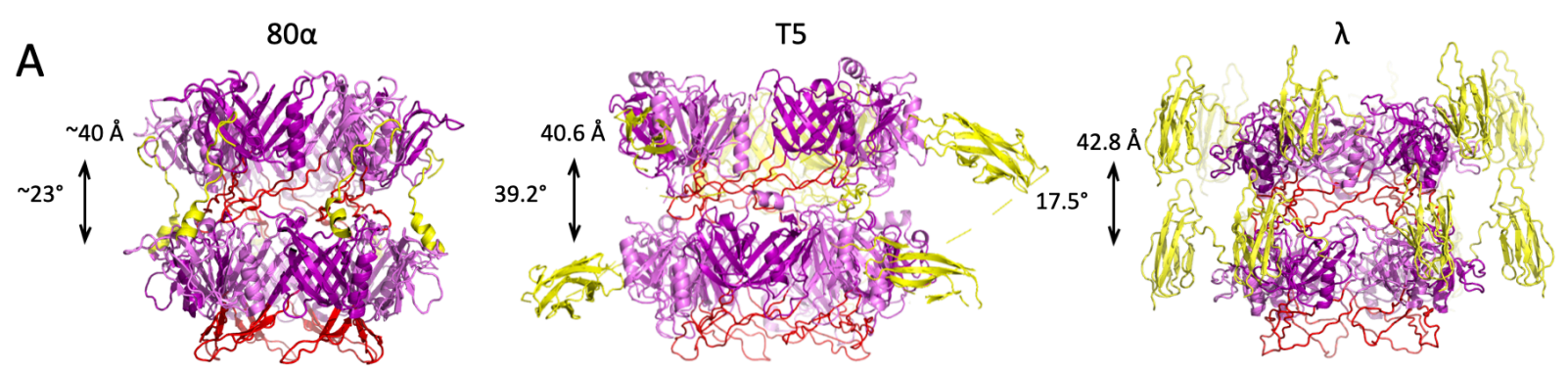

B
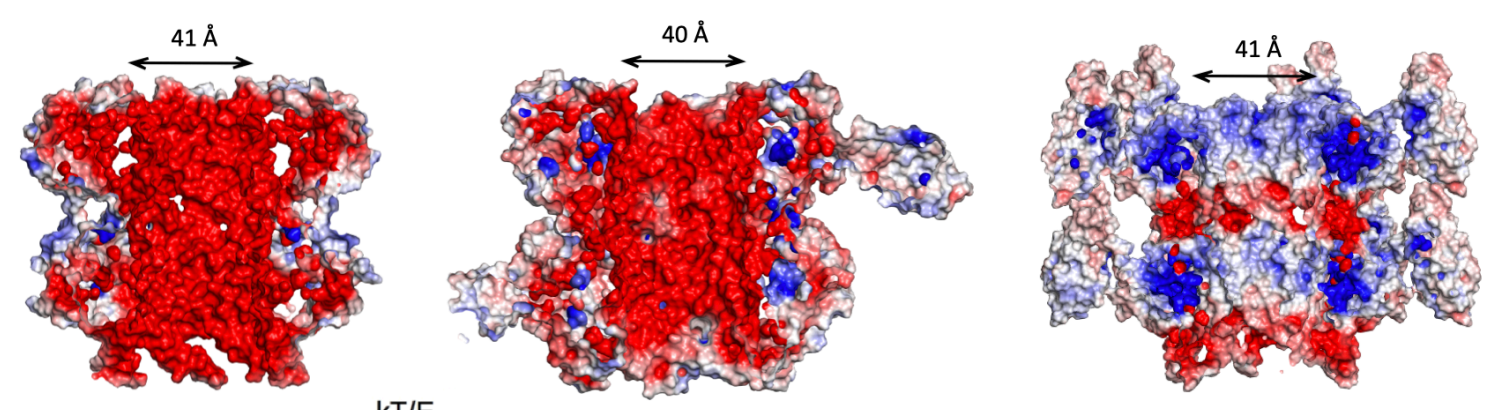

\begin{tabular}{ccc} 
& \multicolumn{2}{c}{$k T / E$} \\
-5 & 0 & 5
\end{tabular}

Figure 3: A. Side views of known tube assembly of siphophages (TTP). Helical rise and twist are indicated. $80 \alpha$ tube structure was solved as part of the tip, thus helical parameters were not refined through the reconstruction process; the two rings are not as fully reconstructed and have slightly different conformations. The large loop is represented in red while C-terminal structures are in yellow. B. Electrostatic charge distribution of the lumen of siphophages tubes. $80 \alpha$ and T5 are vastly negative; this was proposed to smooth DNA transfer through the tube during viral entry. In contrast, $\lambda$ has negatively charged inter-ring loops while most of the inner-tube surface is not. Charge distribution in $\lambda$ tube was proposed to be involved in the assembly/pH sensitive disassembly of the tube of $\lambda[13]$. 
A
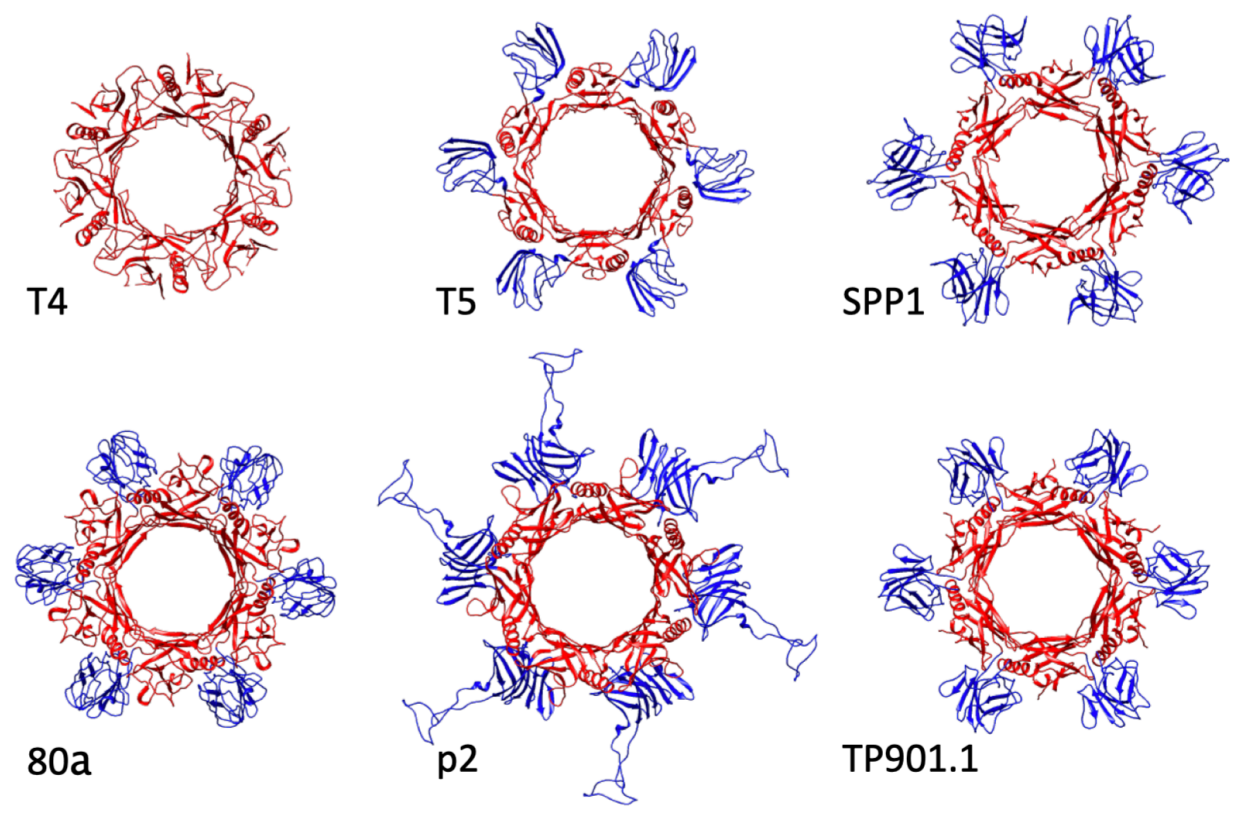

B
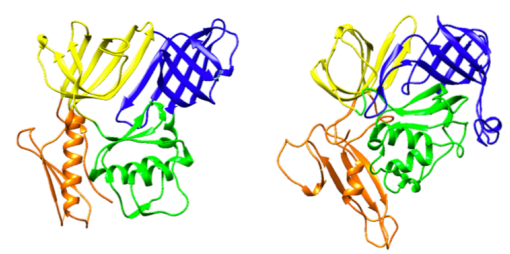

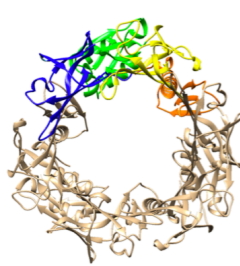

$\mathrm{Mu}$

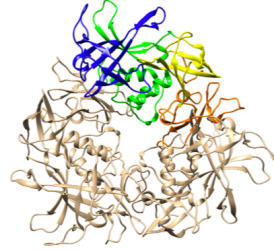

p2
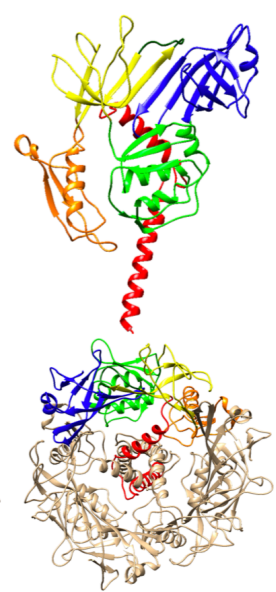

$80 \mathrm{a}$

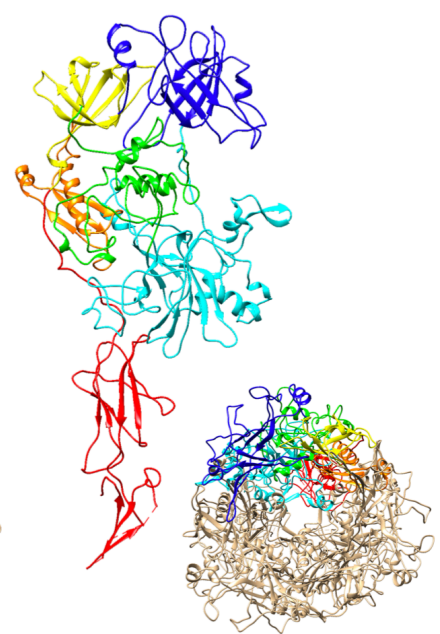

T5

Figure 4: Structural homologies of DTPs and BHPs. A. Top views of the DTP hexameric ring of phages T4 (5IV5), T5 (4JMQ), SPP1 (2X8K), 80 $\alpha$ (6V8I), p2 (2WZP) and TP901-1 (4V96). The TTP fold is coloured red and decoration domains are coloured blue. B. Side views of the monomeric (top) and top views (bottom) of the trimeric BHP of phages Mu (1WRU), p2 (2WZP), 80a (6V8I) and T5 (to be published). Hub Domains I-IV are coloured blue, green, yellow and orange, respectively, on one monomer. In T5, the extension of HDII is coloured cyan, and in $80 \alpha$ and T5, C-terminal extensions are coloured red. In 80a-BHP the C-terminal domain, predicted to fold as a lipase domain, is not resolved and in T5, the second fibronectin domain is poorly resolved. 


\section{References}

1.** Veesler D, Cambillau C: A Common Evolutionary Origin for Tailed-Bacteriophage Functional Modules and Bacterial Machineries. Microbiol Mol Biol Rev MMBR 2011, 75:423-433.

A review that first analyses the common folds shared by all phages.

2.** Davidson AR, Cardarelli L, Pell LG, Radford DR, Maxwell KL: Long noncontractile tail machines of bacteriophages. Adv Exp Med Biol 2012, 726:115-142.

Extensive review that summarises many decades worth of research that cover a broad range of topics concerning the non-contractile tails of bacteriophages

3. Auzat I, Petitpas I, Lurz R, Weise F, Tavares P: A touch of glue to complete bacteriophage assembly: the tail-to-head joining protein (THJP) family. Mol Microbiol 2014, 91:1164-1178.

4. Zivanovic Y, Confalonieri F, Ponchon L, Lurz R, Chami M, Flayhan A, Renouard M, Huet A, Decottignies P, Davidson AR, et al.: Insights into bacteriophage T5 structure from analysis of its morphogenesis genes and protein components. $J$ Virol 2014, 88:1162-1174.

5. Iwasaki T, Yamashita E, Nakagawa A, Enomoto A, Tomihara M, Takeda S: Threedimensional structures of bacteriophage neck subunits are shared in Podoviridae, Siphoviridae and Myoviridae. Genes Cells Devoted Mol Cell Mech 2018, 23:528-536.

6.* Chaban Y, Lurz R, Brasilès S, Cornilleau C, Karreman M, Zinn-Justin S, Tavares P, Orlova EV: Structural rearrangements in the phage head-to-tail interface during assembly and infection. Proc Natl Acad Sci U S A 2015, 112:7009-7014.

First structure of the head-to-tail connection of a siphophage, .

7. Fokine A, Zhang Z, Kanamaru S, Bowman VD, Aksyuk AA, Arisaka F, Rao VB, Rossmann MG: The molecular architecture of the bacteriophage T4 neck. J Mol Biol 2013, 425:1731-1744.

8. Lopes A, Tavares P, Petit M-A, Guérois R, Zinn-Justin S: Automated classification of tailed bacteriophages according to their neck organization. BMC Genomics 2014, 15:1027.

9. Tavares P: The Bacteriophage Head-to-Tail Interface. Subcell Biochem 2018, 88:305328.

10. Pell LG, Kanelis V, Donaldson LW, Howell PL, Davidson AR: The phage lambda major tail protein structure reveals a common evolution for long-tailed phages and the type VI bacterial secretion system. Proc Natl Acad Sci U S A 2009, 106:4160-4165.

11. Zinke M, Fricke P, Samson C, Hwang S, Wall JS, Lange S, Zinn-Justin S, Lange A: Bacteriophage Tail-Tube Assembly Studied by Proton-Detected 4D Solid-State NMR. Angew Chem Int Ed Engl 2017, 56:9497-9501.

12.** Arnaud C-A, Effantin G, Vivès C, Engilberge S, Bacia M, Boulanger P, Girard E, Schoehn G, Breyton C: Bacteriophage T5 tail tube structure suggests a trigger mechanism for Siphoviridae DNA ejection. Nat Commun 2017, 8:1953.

First pseudo-atomic structure of the tail tube of a siphophage, before and after interaction with the recpetor.

13. Campbell PL, Duda RL, Nassur J, Conway JF, Huet A: Mobile Loops and Electrostatic Interactions Maintain the Flexible Tail Tube of Bacteriophage Lambda. J Mol Biol 2020, 432:384-395.

14.** Taylor NMI, van Raaij MJ, Leiman PG: Contractile injection systems of bacteriophages and related systems. Mol Microbiol 2018, 108:6-15.

Reviews the similarities and differences between myophages and bacterial phage-derived contractile injection systems.

15. Langlois C, Ramboarina S, Cukkemane A, Auzat I, Chagot B, Gilquin B, Ignatiou A, 
Petitpas I, Kasotakis E, Paternostre M, et al.: Bacteriophage SPP1 tail tube protein selfassembles into $\boldsymbol{\beta}$-structure-rich tubes. J Biol Chem 2015, 290:3836-3849.

16. Špakova A, Šimoliūnas E, Batiuškaitè R, Pajeda S, Meškys R, Petraitytė-Burneikienè R: Self-Assembly of Tail Tube Protein of Bacteriophage vB_EcoS_NBD2 into Extremely Long Polytubes in E. coli and S. cerevisiae. Viruses 2019, 11.

17. Plisson C, White HE, Auzat I, Zafarani A, São-José C, Lhuillier S, Tavares P, Orlova EV: Structure of bacteriophage SPP1 tail reveals trigger for DNA ejection. EMBO J 2007, 26:3720-3728.

18. Fraser JS, Maxwell KL, Davidson AR: Immunoglobulin-like domains on bacteriophage: weapons of modest damage? Curr Opin Microbiol 2007, 10:382-387.

19. Auzat I, Dröge A, Weise F, Lurz R, Tavares P: Origin and function of the two major tail proteins of bacteriophage SPP1. Mol Microbiol 2008, 70:557-569.

20. Fraga H, Arnaud C-A, Gauto DF, Audin MJC, Kurauskas V, Macek P, Krichel C, Guan J-Y, Boisbouvier J, Sprangers R, et al:: Solid-state NMR H-N-(C)-H and H-N-C-C 3D/4D correlation experiments for resonance assignment of large proteins. Chemphyschem Eur $J$ Chem Phys Phys Chem 2017, doi:10.1002/cphc.201700572.

21. Kizziah JL, Manning KA, Dearborn AD, Dokland T: Structure of the host cell recognition and penetration machinery of a Staphylococcus aureus bacteriophage. PLoS Pathog 2020, 16:e1008314.

22.** Katsura I: Determination of bacteriophage lambda tail length by a protein ruler. Nature 1987, 327:73-75.

Elegant demonstration of the role of the Tape Measure Protein in determining the length of phage tails.

23. Mahony J, Alqarni M, Stockdale S, Spinelli S, Feyereisen M, Cambillau C, Sinderen D van: Functional and structural dissection of the tape measure protein of lactococcal phage TP901-1. Sci Rep 2016, 6:36667.

24. Boulanger P, Jacquot P, Plançon L, Chami M, Engel A, Parquet C, Herbeuval C, Letellier L: Phage T5 straight tail fiber is a multifunctional protein acting as a tape measure and carrying fusogenic and muralytic activities. J Biol Chem 2008, 286:13556-13564.

25. Tsui LC, Hendrix RW: Proteolytic processing of phage lambda tail protein gpH: timing of the cleavage. Virology 1983, 125:257-264.

26. $\mathrm{Xu} \mathrm{J}$, Hendrix $\mathrm{RW}$, Duda $\mathrm{RL}$ : A balanced ratio of proteins from gene $\mathbf{G}$ and frameshift-extended gene GT is required for phage lambda tail assembly. $J$ Mol Biol 2013, 425:3476-3487.

27. Pell LG, Cumby N, Clark TE, Tuite A, Battaile KP, Edwards AM, Chirgadze NY, Davidson AR, Maxwell KL: A conserved spiral structure for highly diverged phage tail assembly chaperones. $J$ Mol Biol 2013, 425:2436-2449.

28.** $\mathrm{Xu}$ J, Hendrix RW, Duda RL: Chaperone-protein interactions that mediate assembly of the bacteriophage lambda tail to the correct length. J Mol Biol 2014, 426:1004-1018.

Both Xu et al. (2013 and 2014) papers dissect the role of both tail chaperones in tail assembly and TTP polymerisation.

29. Cumby N, Reimer K, Mengin-Lecreulx D, Davidson AR, Maxwell KL: The phage tail tape measure protein, an inner membrane protein and a periplasmic chaperone play connected roles in the genome injection process of E. coli phage HK97. Mol Microbiol 2015, 96:437-447.

30. Bebeacua C, Lorenzo Fajardo JC, Blangy S, Spinelli S, Bollmann S, Neve H, Cambillau C, Heller KJ: X-ray structure of a superinfection exclusion lipoprotein from phage TP-J34 and identification of the tape measure protein as its target. Mol Microbiol 2013, 89:152-165.

31. Bebeacua C, Lai L, Vegge CS, Brøndsted L, van Heel M, Veesler D, Cambillau C: 
Visualizing a complete Siphoviridae member by single-particle electron microscopy: the structure of lactococcal phage TP901-1. J Virol 2013, 87:1061-1068.

32. Legrand P, Collins B, Blangy S, Murphy J, Spinelli S, Gutierrez C, Richet N, Kellenberger C, Desmyter A, Mahony J, et al:: The Atomic Structure of the Phage Tuc2009 Baseplate Tripod Suggests that Host Recognition Involves Two Different Carbohydrate Binding Modules. mBio 2016, 7:e1781-01715.

33. Goulet A, Lai-Kee-Him J, Veesler D, Auzat I, Robin G, Shepherd DA, Ashcroft AE, Richard E, Lichière J, Tavares P, et al.: The Opening of the SPP1 Bacteriophage Tail, a Prevalent Mechanism in Gram-positive-infecting Siphophages. J Biol Chem 2011, 286:25397-25405.

34. Veesler D, Spinelli S, Mahony J, Lichière J, Blangy S, Bricogne G, Legrand P, OrtizLombardia M, Campanacci V, van Sinderen D, et al.: Structure of the phage TP901-1 1.8 MDa baseplate suggests an alternative host adhesion mechanism. Proc Natl Acad Sci US A 2012, 109:8954-8958.

35.** Sciara G, Bebeacua C, Bron P, Tremblay D, Ortiz-Lombardia M, Lichière J, van Heel M, Campanacci V, Moineau S, Cambillau C: Structure of lactococcal phage p2 baseplate and its mechanism of activation. Proc Natl Acad Sci U S A 2010, 107:6852-6857.

First atomic structure of an over-expressed siphophage baseplate, compared to a low-resolution envelop of the in phage baseplate. Structure comparison the baseplate with and without $\mathrm{Ca}^{2+}$ mandatory for infection- reveal the activation mechanism of phage $\mathrm{p} 2$.

36. Shepherd DA, Veesler D, Lichière J, Ashcroft AE, Cambillau C: Unraveling lactococcal phage baseplate assembly by mass spectrometry. Mol Cell Proteomics MCP 2011, 10:M111.009787.

37. Stockdale SR, Collins B, Spinelli S, Douillard FP, Mahony J, Cambillau C, van Sinderen D: Structure and Assembly of TP901-1 Virion Unveiled by Mutagenesis. PloS One 2015, 10:e0131676.

38. Mahony J, Stockdale SR, Collins B, Spinelli S, Douillard FP, Cambillau C, van Sinderen D: Lactococcus lactis phage TP901-1 as a model for Siphoviridae virion assembly. Bacteriophage 2016, 6:e1123795.

39. Flayhan A, Vellieux FMD, Lurz R, Maury O, Contreras-Martel C, Girard E, Boulanger P, Breyton C: Crystal Structure of pb9, the Distal Tail Protein of Bacteriophage T5: a Conserved Structural Motif among All Siphophages. J Virol 2014, 88:820-828.

40. Wang Z, Hardies SC, Fokine A, Klose T, Jiang W, Cho BC, Rossmann MG: Structure of the Marine Siphovirus TW1: Evolution of Capsid-Stabilizing Proteins and Tail Spikes. Struct Lond Engl 1993 2018, 26:238-248.e3.

41.** Spinelli S, Veesler D, Bebeacua C, Cambillau C: Structures and host-adhesion mechanisms of lactococcal siphophages. Front Microbiol 2014, 5:3.

Comprehensive review comparing the different lactococcal BRPs, baseplate structures, activation and adhesion mechanisms.

42. Hayes S, Vincentelli R, Mahony J, Nauta A, Ramond L, Lugli GA, Ventura M, van Sinderen D, Cambillau C: Functional carbohydrate binding modules identified in evolved dits from siphophages infecting various Gram-positive bacteria. Mol Microbiol 2018, 110:777-795.

43. Dieterle ME, Fina Martin J, Durán R, Nemirovsky SI, Sanchez Rivas C, Bowman C, Russell D, Hatfull GF, Cambillau C, Piuri M: Characterization of prophages containing "evolved" Dit/Tal modules in the genome of Lactobacillus casei BL23. Appl Microbiol Biotechnol 2016, 100:9201-9215.

44. Dieterle M-E, Spinelli S, Sadovskaya I, Piuri M, Cambillau C: Evolved distal tail carbohydrate binding modules of Lactobacillus phage J-1: a novel type of anti-receptor widespread among lactic acid bacteria phages. Mol Microbiol 2017, doi:10.1111/mmi.13649. 
45.** Kanamaru S, Leiman PG, Kostyuchenko VA, Chipman PR, Mesyanzhinov VV, Arisaka F, Rossmann MG: Structure of the cell-puncturing device of bacteriophage T4. Nature 2002, 415:553-557.

First structure of a cell-puncturing device, that of T4, describing, among others, the 6-to-3 fold adaptor and the first "TTP-type" fold in the BHP gp27.

46.* Vinga I, Baptista C, Auzat I, Petipas I, Lurz R, Tavares P, Santos MA, São-José C: Role of bacteriophage SPP1 tail spike protein gp21 on host cell receptor binding and trigger of phage DNA ejection. Mol Microbiol 2012, 83:289-303.

Paper dissecting the role of the central fibre protein of phage SPP1.

47. Bebeacua C, Tremblay D, Farenc C, Chapot-Chartier M-P, Sadovskaya I, van Heel M, Veesler D, Moineau S, Cambillau C: Structure, adsorption to host, and infection mechanism of virulent lactococcal phage p2. J Virol 2013, 87:12302-12312.

48. Stockdale SR, Mahony J, Courtin P, Chapot-Chartier M-P, van Pijkeren J-P, Britton RA, Neve H, Heller KJ, Aideh B, Vogensen FK, et al.: The lactococcal phages Tuc2009 and TP901-1 incorporate two alternate forms of their tail fiber into their virions for infection specialization. $J$ Biol Chem 2013, 288:5581-5590.

49. Spinelli S, Bebeacua C, Orlov I, Tremblay D, Klaholz BP, Moineau S, Cambillau C: Cryo-electron microscopy structure of lactococcal siphophage 1358 virion. J Virol 2014, 88:8900-8910.

50. Mahony J, van Sinderen D: Gram-positive phage-host interactions. Front Microbiol 2015, 6:61.

51.* Mahony J, Cambillau C, van Sinderen D: Host recognition by lactic acid bacterial phages. FEMS Microbiol Rev 2017, 41:S16-S26.

Comprehensive review on the process of haw lactococcal phages recognise and bind their host, addressed by genomic/molecular and structural analyses combined with host cell wall biochemical studies.

52. Dunne M, Hupfeld M, Klumpp J, Loessner MJ: Molecular Basis of Bacterial Host Interactions by Gram-Positive Targeting Bacteriophages. Viruses 2018, 10.

53. Nobrega FL, Vlot M, de Jonge PA, Dreesens LL, Beaumont HJE, Lavigne R, Dutilh BE, Brouns SJJ: Targeting mechanisms of tailed bacteriophages. Nat Rev Microbiol 2018, 16:760-773.

54. Aksyuk AA, Bowman VD, Kaufmann B, Fields C, Klose T, Holdaway HA, Fischetti VA, Rossmann MG: Structural investigations of a Podoviridae streptococcus phage C1, implications for the mechanism of viral entry. Proc Natl Acad Sci 2012, 109:14001-14006.

55. Fokine A, Rossmann MG: Common Evolutionary Origin of Procapsid Proteases, Phage Tail Tubes, and Tubes of Bacterial Type VI Secretion Systems. Struct Lond Engl 1993 2016, 24:1928-1935.

56.** $\mathrm{Hu} \mathrm{B}$, Margolin $\mathrm{W}$, Molineux IJ, Liu J: Structural remodeling of bacteriophage T4 and host membranes during infection initiation. Proc Natl Acad Sci U S A 2015, 112:E49194928.

Cryo-electron tomography of mini-skinny-coli cells infected with myophage T4, showing different infecting intermediates in cell wall perforation.

57. Wang C, Tu J, Liu J, Molineux IJ: Structural dynamics of bacteriophage P22 infection initiation revealed by cryo-electron tomography. Nat Microbiol 2019, doi:10.1038/s41564019-0403-z 